\title{
ANÁLISE DAS RESPOSTAS AGUDAS DA FREQUÊNCIA CARDÍACA, PRESSÃO ARTERIAL E DUPLO PRODUTO NO TREINAMENTO RESISTIDO COM DIFERENTES EXERCÍCIOS E INTENSIDADES ANALYSIS OF ACUTE RESPONSES OF HEART RATE, BLOOD PRESSURE AND DOUBLE PRODUCT ON RESISTANCE TRAINING WITH DIFFERENT EXERCISES AND INTENSITIES
}

\author{
Diogo Correia Cardozo', Marcelo Ricardo Cabral Dias ${ }^{1}$ \\ 'Laboratório de Fisiologia do Exercício e Avaliação Morfo-Funcional da Faculdade Metodista Granbery - Juiz de Fora (MG), Brasil.
}

\section{RESUMO}

Introdução: Alguns indicadores de estresse cardiovascular fornecem importantes informações para monitorar o esforço durante a prática de exercício resistido. Contudo, não existe um consenso com relação ao tipo de exercício e intensidade de um mesmo grupo muscular. Objetivo: Analisar as respostas da frequência cardíaca, pressão arterial e do duplo produto em diferentes intensidades de exercício resistido uni e multiarticular. Materiais e Métodos: Foram recrutados 11 universitários normotensos, praticantes de treinamento resistido $(22,2 \pm 2,8$ anos de idade; $171,2 \pm 9,4 \mathrm{~cm}$ de estatura; $68,0 \pm 12,6 \mathrm{~kg}$ de peso corporal; $23,0 \pm 2,4$ índice de massa corporal). Estes realizaram 3 séries com 60 e $80 \%$ de uma repetição máxima nos exercícios de voador e supino horizontal até a falha concêntrica. A pressão arterial e a frequência cardíaca foram obtidas em repouso e imediatamente após cada série dos exercícios. Resultados: Os resultados indicam que o tipo de exercício não influencia nas respostas cardiovasculares. Entretanto, com relação à intensidade, somente foi observado diferença estatística para o exercício voador na intensidade de 60\% de 1RM (uma repetição máxima), com maiores valores encontrados quando comparado à intensidade de $80 \%$. Conclusão: Os resultados do presente estudo indicam que o tipo de exercício realizado (uni ou multiarticular) não influencia em elevar as respostas cardiovasculares agudas ao esforço em jovens normotensos. Contudo, com relação à intensidade do exercício, foi observado que a mesma, exerce maior estresse cardiovascular apenas para o exercício uniarticular voador. Fato este, que deve ser considerado para prescrição de populações especiais.

Palavras-chave: treinamento de resistência; pressão arterial; frequência cardíaca.

\section{ABSTRACT}

Introduction: Some cardiovascular stress indicators provide important information to monitor the effort while practicing resistance exercise. However, there is no consensus regarding the type and intensity of exercise on the same muscle group. Objective: To analyze heart rate, blood pressure and rate-pressure product at different intensities of uni-and multi-joint resistance exercise. Materials and Methods: We recruited 11 normotensive university students who practice resistance training $(22.2 \pm 2.8$ years old, $171.2 \pm 9.4 \mathrm{~cm}$ height, $68.0 \pm 12.6 \mathrm{~kg}$ body weight, $23.0 \pm 2.4$ body mass index). They performed 3 sets of 60 to $80 \%$ of one maximum repetition in flying exercises and bench press until concentric failure. Blood pressure and heart rate were obtained at rest and immediately after each set of exercises. Results: The results indicate that the type of exercise has no effect on cardiovascular responses. However, statistical difference was only observed in flying exercise at 60\% $1 \mathrm{MR}$ (one maximum repetition) intensity, with higher values found when they were compared to $80 \%$ intensity. Conclusion: The results of this study indicate that the type of exercise performed (single or multi-joint resistance exercise) has no effect on cardiovascular responses to acute stress in young normotensive subjects. However, regarding the exercise intensity, higher cardiovascular stress was only observed in the uni-joint flying exercise. This fact can be considered in prescribing to special populations.

Keywords: resistance training; arterial pressure; heart rate. 


\section{INTRODUÇÃO}

Atualmente, os exercícios resistidos vêm sendo indicados como possibilidade de intervenção para o controle da pressão arterial e doenças cardíacas ${ }^{1,2}$. Contudo, visando à segurança durante a sua pratica, os exercícios resistidos devem requerer alguns cuidados com relação a sua prescrição. Desta forma, alguns indicadores de estresse cardiovascular podem fornecer importantes informações para monitorar o esforço durante a prática dos exercícios resistidos ${ }^{3-5}$. As respostas hemodinâmicas parecem variar com os diferentes protocolos empregados de exercícios resistidos $^{5,6}$. Algumas pesquisas têm verificado que a intensidade exerce influência nas respostas cardiovasculares durante sua execução ${ }^{3,7}$.

Outras variáveis que podem influenciar no comportamento cardiovascular são o intervalo de descanso, o número de séries, o protocolo de treinamento e o tamanho da massa muscular envolvida ${ }^{8-11}$. Com relação ao tamanho do grupamento muscular, é possível pensar que a menor massa muscular solicitada durante o exercício tende a estimular um menor estresse cardíaco, quando comparado ao maior grupamento muscular. Contudo, as pesquisas que analisaram esse tema não demonstraram efeito algum ${ }^{10,11}$.

Desta maneira, a intensidade e a escolha do tipo de exercício, uni ou multiarticular, são de grande importância ao manipular um programa de exercícios resisitidos. As pesquisas que investigaram esse tema compararam grupos musculares discrepantes, não podendo, dessa forma, aplicar os resultados em outros exercícios $^{10-12}$. Portanto, as pesquisas existentes na literatura não fornecem informações com propriedades para que tenhamos um adequado protocolo de treinamento para esses fins.

Sendo assim, é objetivo do presente estudo comparar o comportamento da frequência cardíaca, da pressão arterial e do duplo produto (DP) durante a prática de diferentes exercícios resistidos (uni e multiarticular), com diferentes intensidades, para um mesmo grupamento muscular.

\section{METODOLOGIA}

A amostra foi constituída por 11 indivíduos (8 homens e 3 mulheres), com idade média de 22,2 $\pm 2,8$ anos, massa corporal $68,0 \pm 12,6 \mathrm{~kg}$, estatura $171,2 \pm 9,4 \mathrm{~cm}$ e índice de massa corporal (IMC) de 23,0 2,4 . Como critérios de inclusão, foram considerados: a) todos os voluntários que não possuíam nenhum tipo de limitação muscular e/ou articular, para que não viesse a influenciar na mecânica do movimento; b) todos deveriam ser experientes em TR a pelo menos 6 meses de pratica ininterrupta; c) não poderiam estar sob uso de qualquer substância ou esteroides anabólicos que pudessem interferir nas respostas cardiovasculares.

Antes de iniciar a coleta dos dados deste estudo, os indivíduos leram e assinaram um Termo de Consentimento Livre e Esclarecido, de acordo com a resolução 196/96 do Conselho Nacional de Saúde, para a realização de testes em seres humanos, que explicou especificamente os testes e procedimentos de treinamento que seriam realizadas durante a pesquisa. Todos os procedimentos experimentais foram aprovados pelo Comitê de Ética em Pesquisa da Santa Casa de Misericórdia de Juiz de Fora, Minas Gerais, sob o parecer $n^{\circ}$ 003/2010.

\section{Teste de uma repetição máxima}

Antes dos testes, todos os participantes foram submetidos a uma semana (três dias/semana) de um período de familiarização, na qual executaram os mesmo exercícios usados no teste de uma repetição máxima (1RM), com objetivo de padronizar a técnica dos exercícios. As sessões foram realizadas com 3 séries de 15 repetições, com aproximadamente $40 \%$ da carga a ser utilizada no teste de 1RM. Após o período de familiarização, os participantes realizaram testes de 1RM, conduzidos no mesmo dia para o voador e o supino horizontal (High On, Righetto ${ }^{\circledR}$, Brasil), com 10 minutos de intervalo entre eles, usando uma ordem randomizada por exercício. Um teste e re-teste de 1RM, separados por 72 horas, foram realizados para determinar a confiabilidade da carga. A carga mais pesada alcançada em qualquer um dos testes foi considerada a carga de 1 RM de um determinado exercício.

Para minimizar o erro durante os testes de $1 \mathrm{RM}$, as seguintes estratégias foram adotadas ${ }^{13}$ : os participantes receberam instruções padronizadas sobre o procedimento de teste e sobre a técnica dos exercícios específicos; e um incentivo verbal foi fornecido durante o procedimento de teste. A carga de 1RM foi determinada em até 5 tentativas, com um intervalo de descanso de 5 minutos entre as tentativas e um período de recuperação de 10 minutos foi permitido antes do início dos testes de 1RM do próximo exercício.

\section{Procedimentos experimentais}

Para a realização dos procedimentos, foi recomendado aos indivíduos que não utilizassem do consumo de cafeína e álcool, e não realizassem nenhuma atividade de força nos grupamentos musculares envolvidos no estudo, pelo período de 24 horas antes de cada sessão de teste. 
Após obter as cargas de 1RM, os indivíduos foram submetidos a um aquecimento específico de 20 repetições com $40 \%$ de 1 RM. Posteriormente, todos realizaram 3 séries até a falha concêntrica com 60 e $80 \%$ de 1 RM, em dias distintos e de forma randomizada (indivíduo $x$ carga $\mathrm{x}$ exercício). Foi obedecido um intervalo entre as séries de dois minutos de recuperação.

\section{Medidas da frequência cardíaca e pressão arterial}

Ao chegar ao local dos testes, o indivíduo sentava-se em uma cadeira onde permanecia por 15 minutos em repouso, e assim eram aferidos os valores da pressão arterial $(\mathrm{PA})$ e frequência cardíaca $(\mathrm{FC})$.

Para a medida da PA, foi considerado como valor sistólico a primeira fase do som de Korotkoff e como valor diastólico, a quarta fase. Para a FC, foi utilizado o maior valor registrado na realização do exercício ou momentos após seu término, devido ao tempo necessário para o monitor realizar a leitura correta. Solicitou-se aos avaliados evitarem a Manobra de Valsalva durante a execução dos exercícios.

As medidas da FC foram feitas através de um frequencímetro Polar FS2 (Finlândia). Nas medidas da PA utilizamos o aparelho esfigmomanômetro aneroide Kole (Brasil), sempre localizado no braço direito, em que foi considerado um intervalo de $2-2 \mathrm{mmHg}$. Foi utilizado o teste $t$ de Student pareado, para efeito de comparação entre os valores encontrados nas variáveis fisiológicas. A análise estatística teve como critério de significância $p<0,05$.

\section{RESULTADOS}

Para as medidas de frequência cardíaca, foi verificada diferença significativa dos valores do repouso, com os valores das três séries em ambos exercícios e intensidades. Quando analisadas as respostas da intensidade, somente a primeira série do exercício voador aplicado com intensidade correspondente a $60 \%$ foi diferente da intensidade do mesmo exercício com $80 \%$ de 1 RM. Entre os exercícios não foi verificada diferença estatística (Figura 1).

Para a pressão arterial sistólica (PAS), foi observada diferença significativa nos valores de repouso, com os valores encontrados durante a execução das três séries em ambos os exercícios e intensidades. Não houve diferença entre os exercícios e as intensidades analisadas (Figura 2).

Para as medidas da pressão arterial diastólica, foi verificada diferença significativa dos valores de repouso do exercício voador, com intensidade de $60 \%$ de 1 RM com a segunda e terceira série. Para o exercício voador com intensidade de $80 \%$, não foi verificada diferença dos valores de repouso com as demais séries. Para o exercício supino, a intensidade de $60 \%$ apresentou diferença significativa do repouso com a segunda e terceira série. Para a intensidade de $80 \%$ do exercício supino, as medidas de repouso somente foram diferentes na

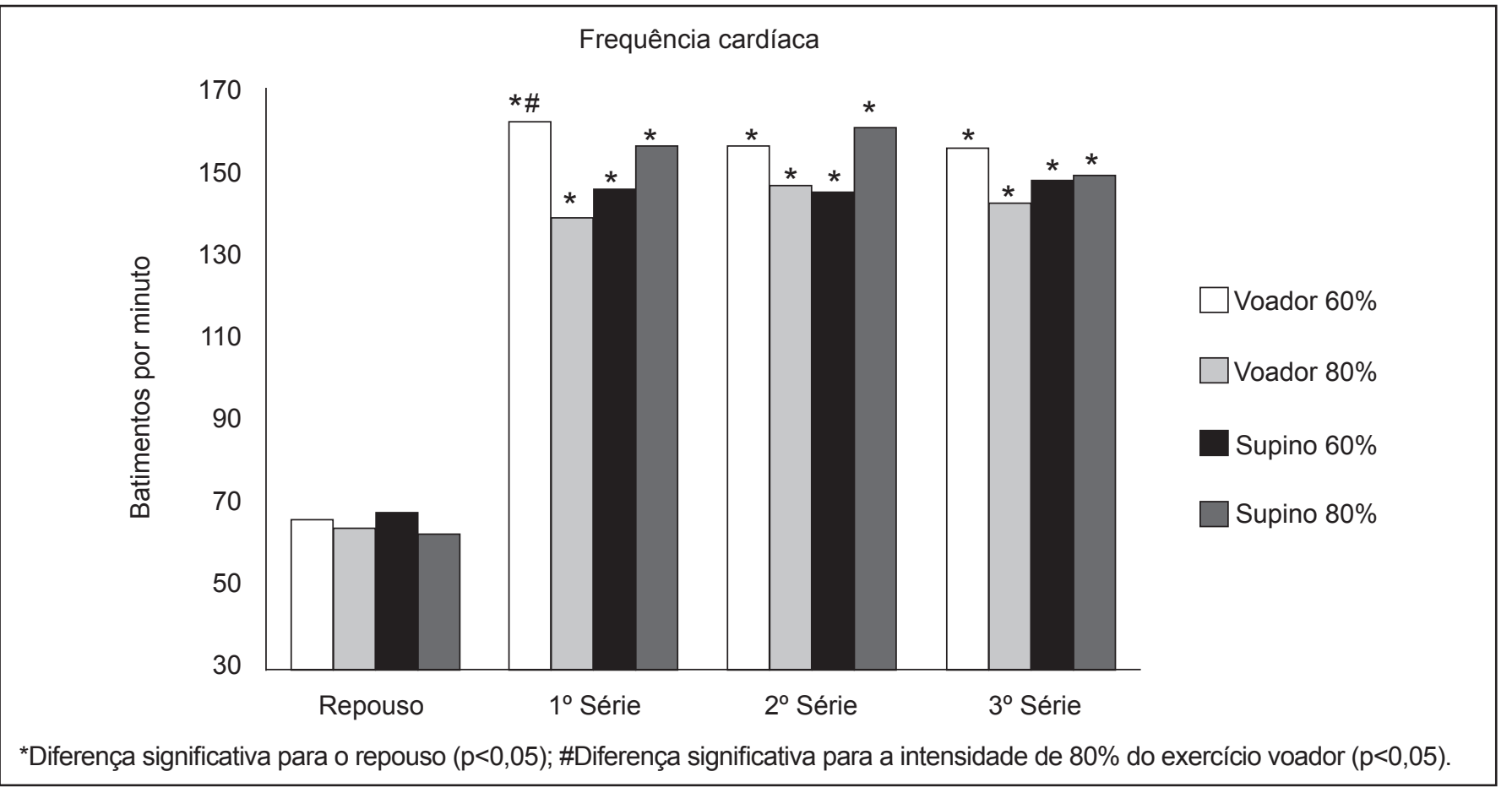

Figura 1: Respostas da frequência cardíaca. 
terceira série. Não foram verificadas diferenças entre os exercícios e as intensidades (Figura 3 ).

A pressão arterial média (PAM) de repouso foi diferente na primeira série do exercício voador com $60 \%$.
$\mathrm{Na}$ intensidade de $80 \%$ do mesmo exercício não foi observada nenhuma diferença. Para o exercício de supino não foi observada nenhuma diferença estatística. Com relação aos exercícios e as intensidades, não foi observada nenhuma diferença (Figura 4).

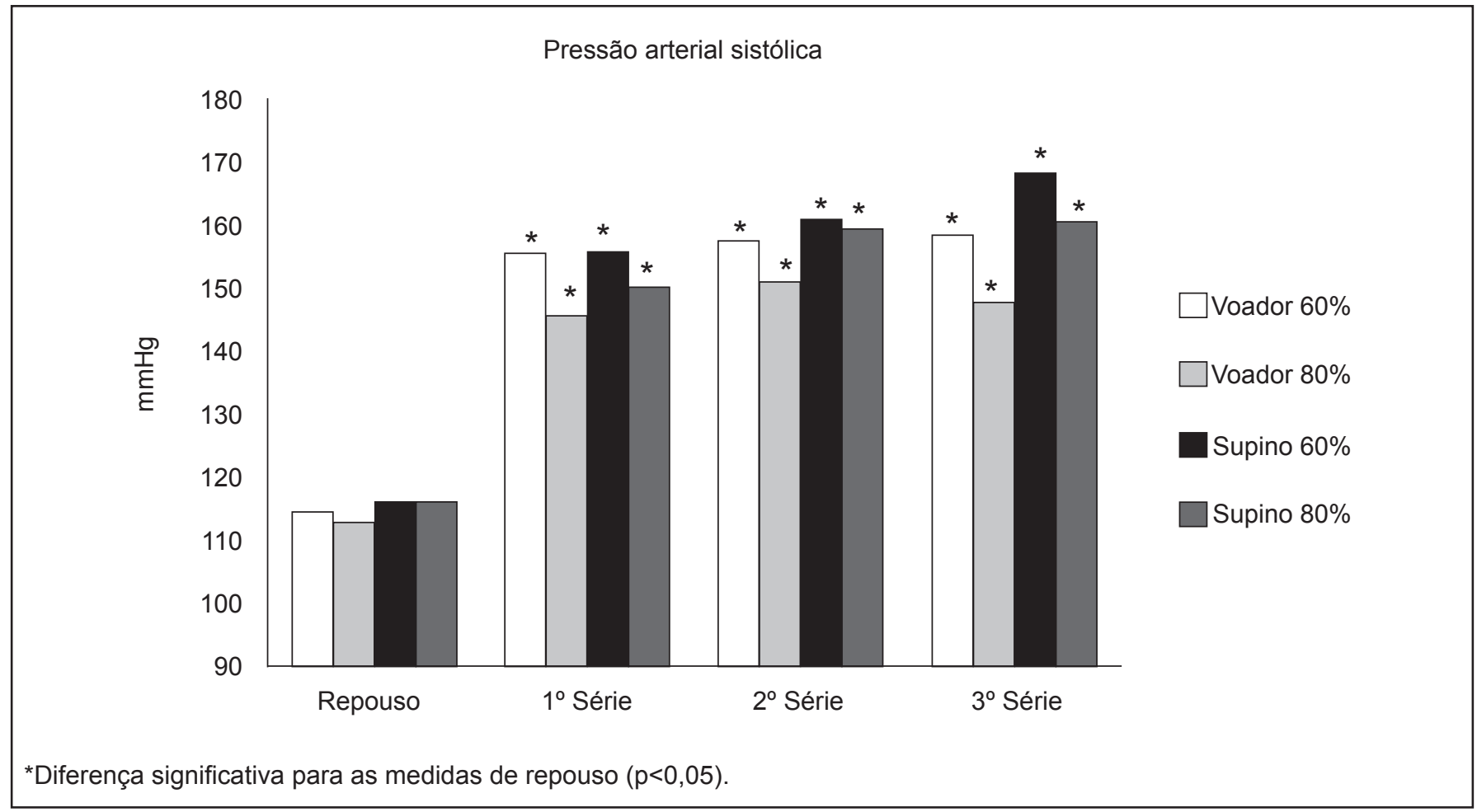

Figura 2: Respostas da pressão arterial sistólica.

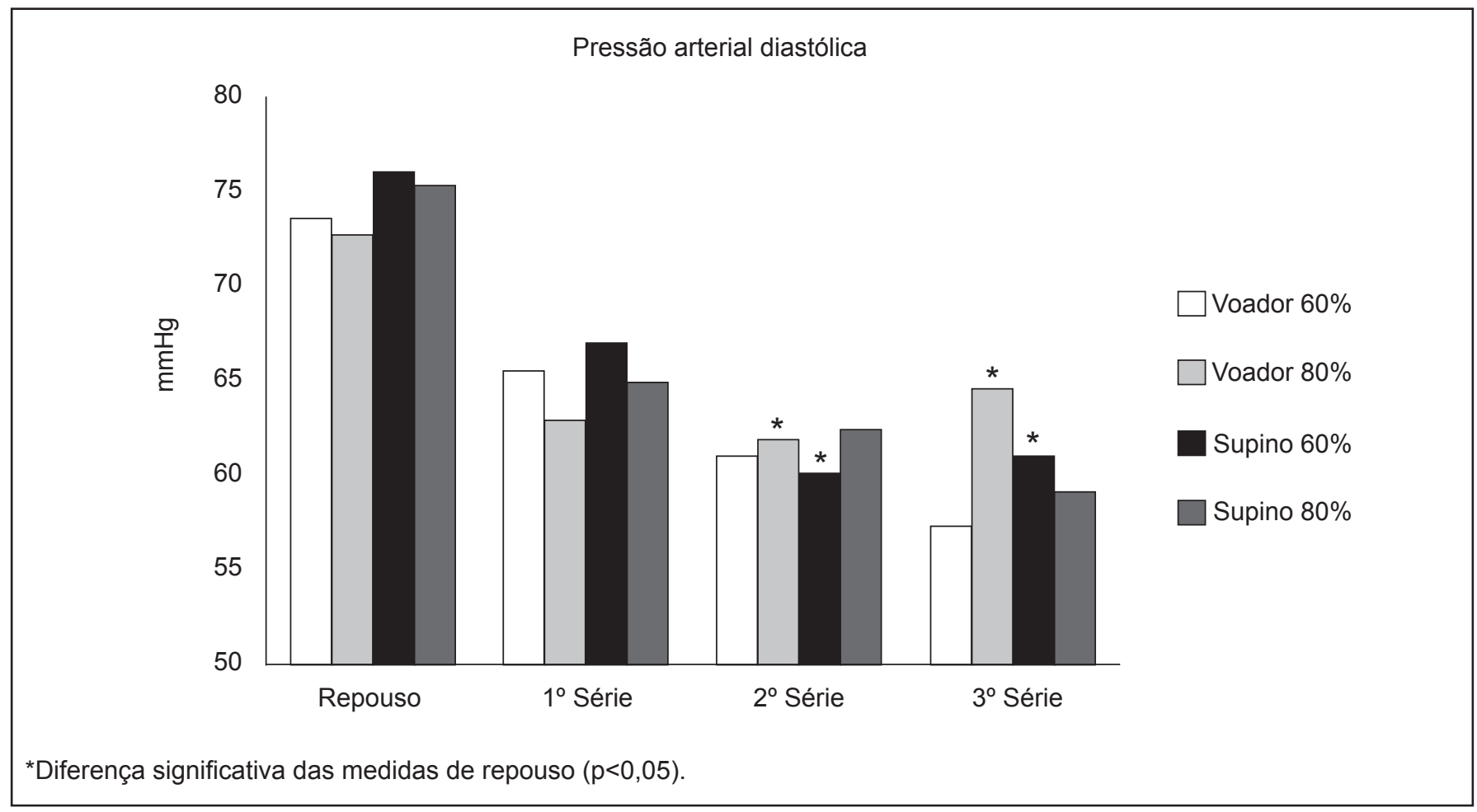

Figura 3: Respostas da pressão arterial diastólica. 
O duplo produto (DP) se apresentou diferente dos valores de repouso com a execução das três séries em ambos os exercícios e intensidades. Analisando o efeito da intensidade de cada exercício, o DP foi diferente apenas no exercício voador com $60 \%$ na primeira e terceira série, quando comparado à intensidade de $80 \%$. Não foram observadas diferenças entre os exercícios (Figura 5).

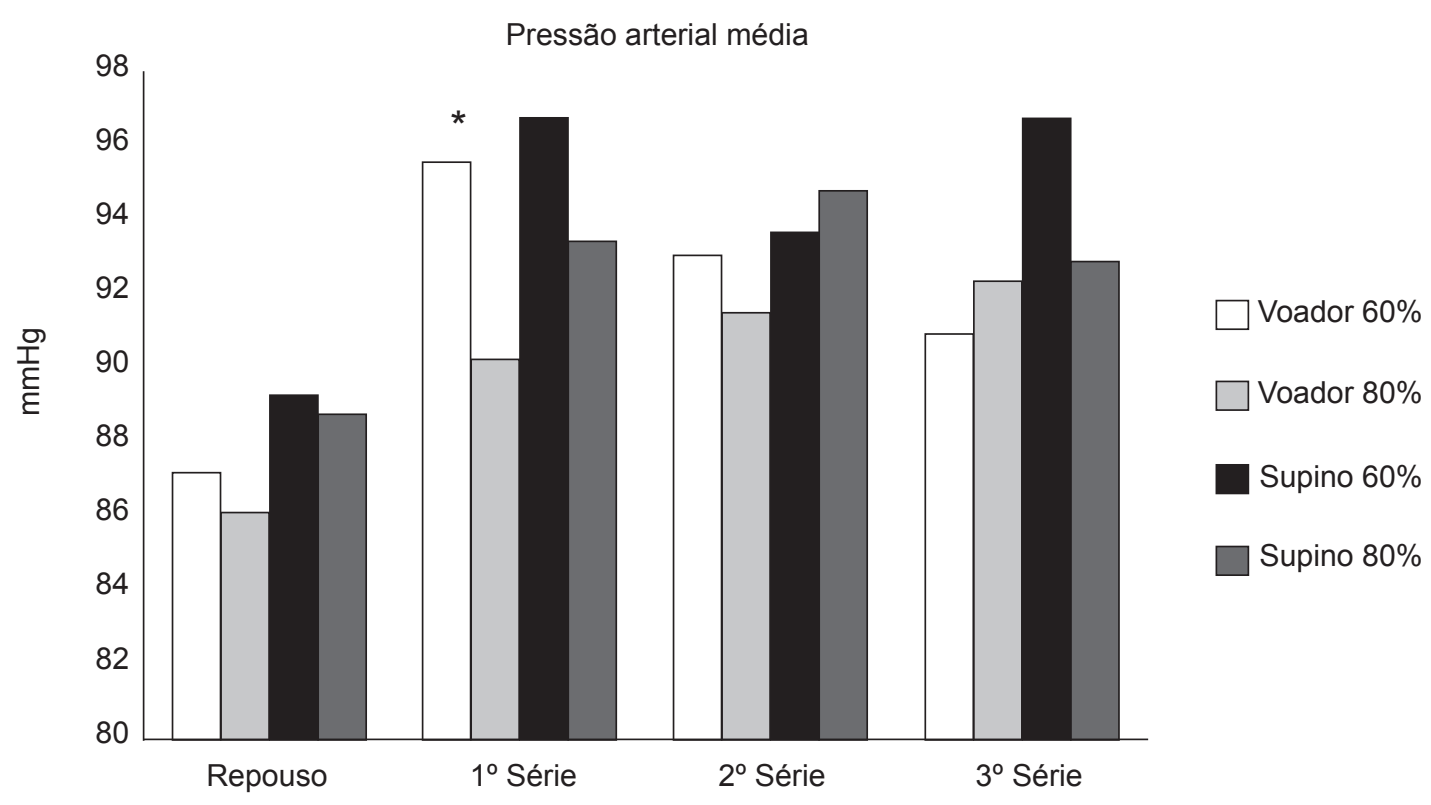

*Diferença significativa das medidas de repouso $(p<0,05)$

Figura 4: Respostas da pressão arterial média.

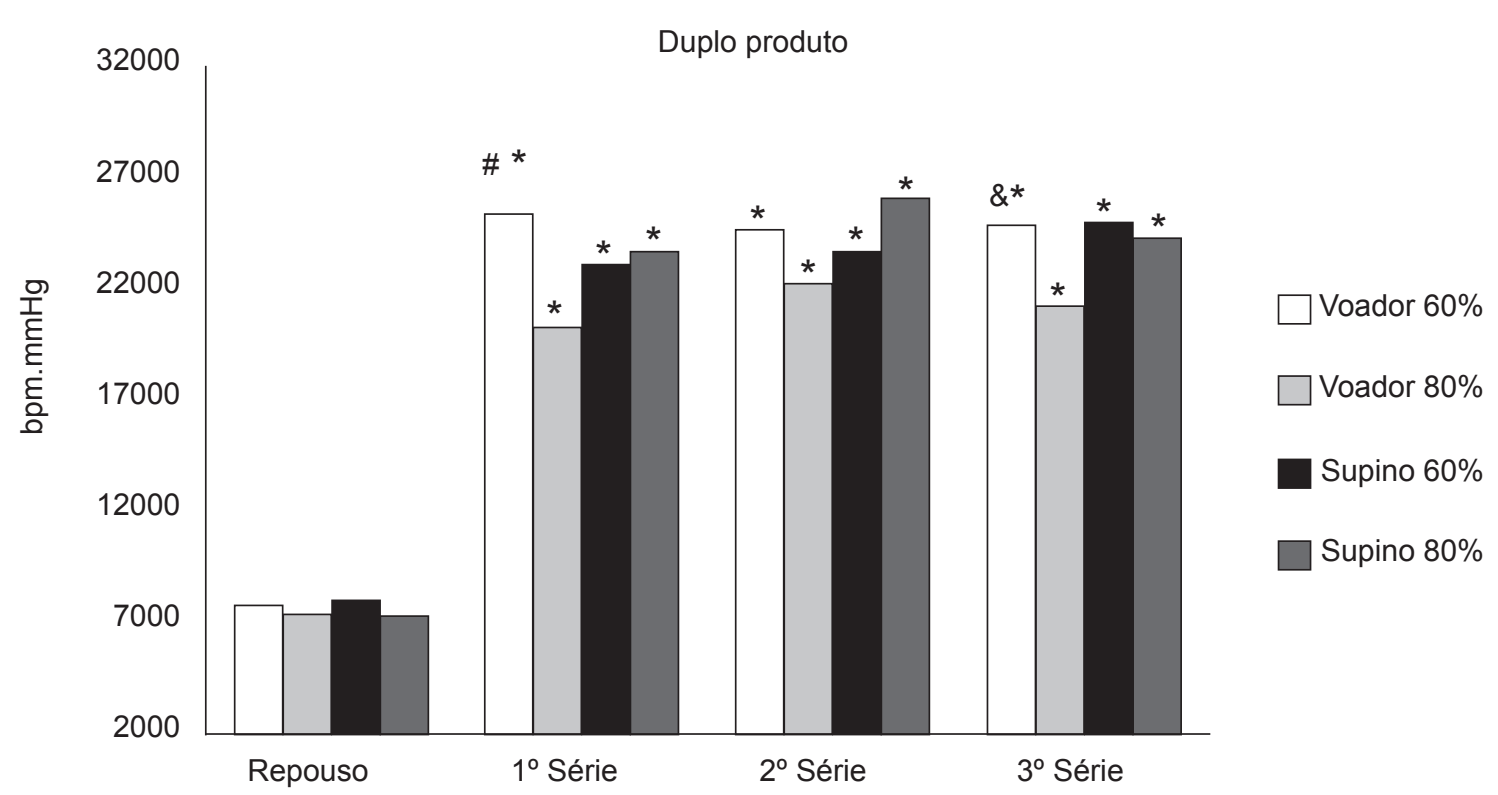

*Diferença significativa para as medidas de repouso $(p<0,05)$; \#Diferença significativa da intensidade do voador na primeira série $(p<0,05)$; \&Diferença significativa da intensidade do exercício voador para a terceira série $(p<0,05)$.

Figura 5: Respostas do duplo produto. 


\section{DISCUSSÃO}

O presente estudo teve como objetivo analisar as respostas cardiovasculares durante diferentes exercícios e intensidades no treinamento resistido. Os resultados demonstraram que as variáveis da FC, PAS, PAM e DP tendem a aumentar significativamente em relação ao momento pré-exercício. Como este estudo também teve como meta investigar duas diferentes intensidades (60 e $80 \%$ de $1 \mathrm{RM}$ ), foi aplicado o protocolo de repetições máximas. Desta forma, e possível que essas respostas sejam devido a ajustes fisiológicos como o aumento da liberação de metabólitos na musculatura ativa. Essas substâncias ativam receptores sensíveis às alterações químicas, também conhecidos como quimiorrecptores, os quais fazem comunicação com o centro de controle cardiovascular, sendo, portanto, responsáveis pelo aumento dessas respostas cardiovasculares durante o exercício ${ }^{14,15}$.

Outro fator a ser considerado é a Manobra de Valsalva, que mesmo tendo sido desaconselhada, devido ao caráter máximo das séries não foi possível controlá-la totalmente.

Os resultados indicam que o tipo de exercício (uni ou muti-articular) não influência nas respostas cardiovasculares durante sua execução, visto que não foram encontradas diferenças significativas entre os exercícios. Esses achados corroboram com outras pesquisas encontradas na literatura. Polito et al. ${ }^{5}$ comparam a extensão do joelho executada de forma uni e bilateralmente em três séries de 12RM e não constataram nenhuma diferença significativa entre as formas de execução. No estudo de Assunção et al. ${ }^{11}$, também não foram observadas diferenças entre os exercícios de rosca bíceps e cadeira extensora. Contudo, nos estudos de MacDougall et al. ${ }^{7} \mathrm{e}$ Gotshal et al. ${ }^{9}$ constataram que o aumento dos níveis de PA se associa ao tamanho da massa muscular recrutada, devido ao aumento da resistência vascular periférica.

O mecanismo fisiológico de ajuste para essas respostas de aumento da PA possa ser devido ao fato de algumas estruturas sensíveis ao aumento da força e da velocidade de movimento, conhecidas também como mecanorrecptores, que, como os quimiorreceptores, informam ao centro de controle cardiovascular para ajustar as respostas cardiovasculares no momento ${ }^{16}$. Outra explicação é que o aumento das unidades motoras recrutadas durante o exercício, ou seja, a maior massa muscular envolvida, desencadeie o aumento dos níveis pressóricos devido à compressão muscular nos leitos vasculares, que consequentemente bloqueiam parcialmente a circulação ${ }^{7}$.
Com relação à intensidade da carga, as diferenças observadas no presente estudo foram para a FC, que apresentou diferença significativa para o exercício voador com os maiores valores para a intensidade de $60 \%$ de $1 \mathrm{RM}$; e a variável do DP, com intensidade também de $60 \%$ de $1 \mathrm{RM}$ do mesmo exercício, que apresentou diferenças significativas na primeira e na terceira série quando comparados à intensidade de $80 \%$.

O DP é uma variável que representa estresse cardiovascular, pois implica na associação dos valores da $\mathrm{FC}$, volume sistólico e débito cardíaco ${ }^{4,6}$. No presente estudo, os valores observados do DP sempre permaneceram abaixo do ponto de corte sugerido para desencadear angina pectoris (30.000 mmHg.bpm), o que implica em um baixo risco ${ }^{17}$. Com relação ao tipo de exercício, não exerceu influência alguma para esta variável (Figura 5). Esses achados corroboram com os estudos de Wilborn et al. ${ }^{12}$, que observaram maiores respostas cardiovasculares na intensidade de $65 \%$ de 1RM e não observaram efeito algum para a posição corporal do exercício. Assim como Miranda et al. ${ }^{18}$, que não verificaram diferenças na posição corporal do exercício nas respostas cardiovasculares.

É importante ressaltar uma limitação metodológica no presente estudo. A PA foi mensurada pelo método auscultatório, tal procedimento subestima os valores durante o exercício ${ }^{9,19}$. Essa resposta ocorre pelo fato desse método não ser sensível em detectar mudanças rápidas nos valores pressóricos. Contudo, permite verificar tendências no comportamento da pressão arterial ${ }^{6,19}$, sendo bem aplicável ${ }^{19}$ e tendo boa correlação com o método fotoplestimográfico, que é considerado o principal método não invasivo $^{19,20}$, justificando então a aplicabilidade do método auscultatório.

\section{CONCLUSÃO}

Os resultados do presente estudo indicam que o tipo de exercício realizado (uni ou multiarticular) não influencia nas respostas cardiovasculares agudas ao esforço em jovens normotensos. Com relação à intensidade do exercício, foi observado, apenas para o exercício uniarticular voador, um maior estresse cardiovascular. Este apresentou maiores valores de FC e DP em algumas séries, quando comparado à intensidade de $80 \%$ do mesmo exercício. Fato este que deve ser considerado para prescrição de grupos que necessitem de cuidados especiais. 


\section{REFERÉNCIAS}

1. Pescatello LS, Franklin BA, Fagard R, Farguhar WB, Kelley GA, Ray CA. American College of Sports Medicine: position stand. Exercise and hypertension. Med Sci Sports Exerc. 2004 mar; 36(3):533-53.

2. Willians MA, Haskell WL, Ades PA, Amsterdam EA, Bittner V, Franklin BA, Gulanick M, Laing ST, Stewart KJ. Resistence exercise in individuals with and without cardiovascular disease: 2007 update: a scientific statement from the American Heart Association Council on Clinical Cardiology and Council on Nutrition, Physical Activity, and Metabolism. Circulation. 2007 jul; 116(5):572-84.

3. Forjaz CLM, Rezk CC, Melo CM, Santos DA, Teixeira L, Nery SS, Tinuci T. Exercício resistido para o paciente hipertenso: indicação ou contra-indicação. Rev Bras Hipertens. 2003; 10:119-24.

4. Polito MD, Farinatti PTV. Respostas de frequência cardíaca, pressão arterial e duplo-produto ao exercício contra resistência: uma revisão de literatura. Rev Port Ciências Desp. 2003; 3(1):79-91.

5. Polito MD, Simão R, Nóbrega ACL, Farinatti PTV. Pressão arterial, frequência cardíaca e duplo-produto em séries sucessivas do exercício de força com diferentes intervalos de recuperação. Rev Port Ciências Desp. 2004; 4(3):7-15.

6. Veloso U, Monteiro W, Farinatti P. Exercícios contínuos e fracionados provocam respostas cardiovasculares similares em idosas praticantes de ginástica? Rev Bras Med Esporte. 2003 mar/abr; 9(2):78-84.

7. MacDougall JD, Tuxen D, Sale DG, Moroz JR, Sutton JR. Arterial blood pressure response to heavy resistance exercise. J Appl Physiol. 1985 mar; 58(3):785-90.

8. Castinheiras-Neto AG, Costa-Filho IR, Farinatti PTV. Respostas cardiovasculares ao exercício resistido são afetadas pela carga e intervalos entre séries. Arq Bras Cardiol. 2010; 95(4):493-501.

9. Gotshall RW, Gootman J, Byrnes WC, Fleck SJ, Valovich TC. Noninvasive characterization of the blood pressure response to the double-leg press exercise. JEP online.1999; 2(4):1-6.

10. Polito MD, Simão R, Lira VA, Nóbrega ACL, Farinatti PTV. Série fracionada da extensão de joelho proporciona maiores respostas cardiovasculares que séries contínuas. Arq Bras Cardiol. 2008; 90(6):382-7.
11. Assunção W, Daltro $M$, Simão R, Polito $M$, Monteiro W. Respostas cardiovasculares agudas no treinamento de força conduzido em exercícios para grandes e pequenos grupamentos musculares. Rev Bras Med Esporte. 2007 mar/abr; 13(2):118-22.

12. Wilborn C, Greenwood M, Wyatt F, Bowden R, Grose $D$. The effects of exercise intensity and body position on cardiovascular variables during resistance exercise. JEP online. 2004; 7(4):29-36.

13. Simão R, Spineti J, de Salles BF, Matta T, Fernandes L, Fleck SJ, Rhea MR, Strom-Olsen HE. Comparison between nonlinear and linear periodized resistance training: hypertrophic and strength effects. J Strength Cond Res. 2012 maio; 26(5):1389-95.

14. Mitchell JH, Payne FC, Saltin B, Schibye B. The role of muscle mass in the cardiovascular response to static contractions. J Physiol. 1980 dez; 309:45-54.

15. Brum PC, Forjaz CLM, Tinucci T, Negrão CE. Adaptações agudas e crônicas do exercício físico no sistema cardiovascular. Rev Paul Educ Fís. 2004 ago; 18:21-31.

16. Prabhakar NR, Peng YJ. Peripheral chemoreceptors in health and disease. J Appl Physiol. 1985 jan; 96(1):359-66.

17. Leite TC, Farinatti PTV. Estudo da frequência cardíaca, pressão arterial e em exercícios resistidos diversos para grupamentos musculares semelhantes. Rev Bras Fisiol Exerc. 2003; 2(1):29-49.

18. Miranda H, Simão R, Lemos A, Dantas BHA, Baptista LA, Novaes J. Análise da freqüência cardíaca, pressão arterial e duplo-produto em diferentes posições corporais nos exercícios resistidos. Rev Bras Med Esporte. 2005 set/out; 11(5):295-8.

19. Polito MD, Farinatti PTV. Considerações sobre a medida da pressão arterial em exercícios contra-resistência. Rev Bras Med Esporte. 2003 jan/fev; 9(1):25-33.

20. Polito MD, Farinatti PT, Lira VA, Nobrega AC. Blood pressure assessment during resistance exercise: comparison between auscultation and Finapres. Blood Press Monit. 2007 abr; 12(2):81-6.

\section{Endereços para correspondência:}

Diogo Correia Cardozo

dcardozoef@gmail.com
Marcelo Ricardo Cabral Dias

diasmr@gmail.com 